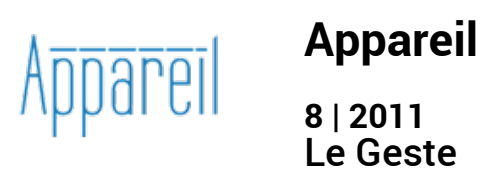

\title{
Glisser - petite topographie du geste en milieu fluide
}

\section{Charlotte Serrus}

\section{(2) OpenEdition}

1 Journals

Édition électronique

URL : http://journals.openedition.org/appareil/1368

DOI : 10.4000/appareil. 1368

ISSN : 2101-0714

Éditeur

MSH Paris Nord

Référence électronique

Charlotte Serrus, « Glisser - petite topographie du geste en milieu fluide », Appareil [En ligne], 8| 2011, mis en ligne le 07 novembre 2011, consulté le 30 juillet 2020. URL : http://journals.openedition.org/ appareil/1368; DOI : https://doi.org/10.4000/appareil.1368

Ce document a été généré automatiquement le 30 juillet 2020.

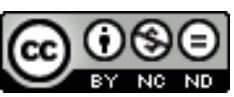

Appareil est mis à disposition selon les termes de la Licence Creative Commons Attribution - Pas d'Utilisation Commerciale - Pas de Modification 4.0 International. 


\title{
Glisser - petite topographie du geste en milieu fluide
}

\author{
Charlotte Serrus
}

J'ai fui en direction d'un monde sans colle, d'un espace Tefal $^{1}$

1 Non pas que la glissade fasse geste au sens fort. Glisser peut être spectaculaire - les parcs d'attraction en témoignent - mais il y a dans ce frottement comme un degré du mineur, une horizontalité constitutive qui le distingue des hautes sphères. La glissade se décline le long des parois lustrées, des textures réfléchissantes; elle se lie à l'inclinaison des sols dans leurs topographies variables, comme l'humour à la doublure des manteaux - chez Deleuze, "l'animal plat des surfaces ${ }^{2}$ ", l'envers poursuivant l'endroit, privé d'ailes comme de profondeur notoire. Glisser s'envisage presque sans coupure, dans une juxtaposition de bouts à bouts spatiaux accolés les uns aux autres et qui permettent les passages. Un tel geste implique un continuum spatio-temporel qui n'est pas nécessairement uniforme, mais dont les prolongations déjouent sans cesse l'arrêt induit par la chute. Tout se passe ici comme si les corps, emportés par les flux, transitaient indéfiniment par quelque escalator aplati, comme on en trouve fréquemment entre deux plateformes dans les centres commerciaux ou les aéroports. En ce sens, non seulement glisser dépossède d'une certaine maîtrise directionnelle, mais procure encore l'expérience paradoxale d'une instabilité sans rupture, dans laquelle la souplesse n'est pas la moindre des qualités requises.

2 Pour ces mêmes raisons, la glissade charrie quantité de valeurs négatives : geste venu d'en bas, qui s'en prend qui plus est à l'équilibre, glisser ne fait pas barrage, et ce manque de solidité apparaît vite comme une facilité, un laisser-aller. Pire qu'un geste avorté, c'est un geste pris dans une pente, semblant par là venir menacer toute possibilité de distanciation. Aussi est-il souvent associé, à juste titre d'ailleurs, aux fluctuations mercantiles et au caractère lisse des modalités de contrôle médiatique : lorsque Hal Foster évoque l'étalage qui prévaut dans le design contemporain, c'est à dessein qu'il emploie le terme "surfer"3. Depuis les sports de glisse jusqu'au surf de l'internaute, des architectures en coques vernies à la promotion généralisée de la 
flexibilité, une partie de l'imaginaire contemporain coïncide, semble-t-il, avec une certaine obsession du lisse. Le geste qui en découle - si tant est qu'il puisse être qualifié de tel - trouve écho dans plusieurs œuvres récentes et interroge son époque, tant au niveau des spatialités et des poétiques instables qu'il dessine, que des modalités de locomotion, d'interaction et de dissidence en milieu fluide. Il met ainsi en jeu, au gré de ses emplois multiples, les possibilités d'investir le lisse aujourd'hui.

\section{La locomotion par flux}

En 2006, Carsten Höller investit le Turbine Hall de la Tate Modern avec un projet intitulé Test Site ${ }^{4}$ : cinq toboggans de verre et d'acier, dont l'ondulation serpentine est rythmée par des anneaux métalliques, sont mis à la disposition des visiteurs; ils desservent chacun des étages du musée pour finir leur course au rez-de-chaussée. Infrastructures euphorisantes à l'esthétique somme toute très pragmatique, ils offrent à la glissade un laboratoire à la fois urbanistique et comportemental : d'une part le toboggan est conçu par l'artiste comme une alternative architecturale aux dispositifs classiques tels que les escaliers ou les ascenseurs, susceptible à terme de faciliter la mobilité des usagers en zone urbaine ${ }^{5}$. Il relève en cela d'une affaire collective qui concerne l'amélioration de la circulation à l'intérieur des bâtiments, autour d'eux, entre eux : rapides, écologiques, simples d'utilisation et fort sympathiques, les toboggans visent à multiplier les interconnexions spatiales, projetant des cités à venir semblables à des "forêts de mangliers ${ }^{6} »$. D'autre part, en termes d'expérience individuelle, la joie enfantine de glisser et le vertige spécifique engendré par la vitesse se lient chez Höller à une disponibilité psychique propice au changement ${ }^{7}$ : une dose quotidienne de glisse, par l'altération perceptive mêlée de plaisir qu'elle suscite, exhorterait chaque utilisateur à remettre en cause son regard sur les choses.

On entrevoit en quoi la glissade peut déjà constituer une expérience potentiellement motrice par sa force de déstabilisation, mais simultanément, le dispositif créé par Höller fait écho aux configurations spécifiques d'une société gouvernée par les flux. Le lâcher prise (décision volontaire, il est vrai) induit par ce transport glissant, en une trajectoire tant unidirectionnelle que sécurisée, correspond aussi bien à un parcours dicté (qui n'est pas sans dialoguer, dans ce contexte, avec l'institution muséale ellemême). Il est d'ailleurs frappant de remarquer que les premiers toboggans réalisés par l'artiste prennent pour titre Valerio ${ }^{8}$ (1998-2000), en référence à une légende urbaine qui relate, vraisemblablement lors d'un concert en Italie, la propagation d'une rumeur sonore : sans que nul ne puisse affirmer avec exactitude qui hurla le premier, le nom de Valerio repris peu à peu par la foule se diffuse par vagues au sein du spectacle. Tour à tour bruissement fascinatoire et outil d'émancipation, le recours à la glissade relève d'une ambivalence constitutive. Par opposition à une avant-garde bien souvent inscrite - au moins discursivement - dans la verticalité temporelle du trancher (la table rase héroïque comme figure de césure absolue), la glissade telle qu'on l'observe aujourd'hui a plus à voir avec une étendue de connections, une mise en réseau excluant les systèmes monadiques, qu'avec le surgissement d'un geste isolé dont la radicalité viendrait fendre l'horizon en deux. Il s'agit moins d'un geste tourné vers l'origine que vers sa rapidité de propagation, sa diffusion par ondes (ce qui implique aussi, faut-il le dire, sa perpétuelle métamorphose au fil des trajectoires). Soit un geste dilué, liquide et polymorphe. 
Car si la glissade fait geste, c'est d'abord dans la mesure où elle entre en résonance étroite avec les formes spatiales et temporelles propres à notre " ère de l'information ", décrite par Manuel Castells ${ }^{9}$ en termes de flux. Sans pour autant s'y réduire, le dispositif proposé par Höller entretient des connivences avec les propriétés du flux ${ }^{10}$ : nous sommes face à une "mise en continuité » qui estompe les démarcations (entre les différents espaces, entre les utilisateurs soumis à un même dispositif), et dont l'écoulement induit une "disponibilité permanente» du service (tout comme les informations se diffusent en boucle). Les statuts et contenus y apparaissent mouvants et difficilement formalisables, dans une prédisposition appuyée pour la mise en relation (les fameux "liens") qui ne se va pas sans dégager une certaine décontraction (chez Höller, la glissade comme instrument de réduction du stress). Derrière cette liberté apparente, le flux témoigne pourtant d'un pouvoir homogénéisant, d'une rare force d'absorption dont l'abondance n'a d'égal que la manière directe qu'il a de se livrer : de ce point de vue, l'un des enjeux du geste de glisser au sein des pratiques artistiques contemporaines consiste à réintroduire des différences dans cette coulée englobante, et à promouvoir un travail de médiation tout en ayant parfois recours aux mêmes dispositifs associatifs et non hiérarchiques qui président au fonctionnement des flux. Si l'état de latence promu par Test Site - le trouble introduit en glissant à l'égard de l' habitus perceptif - est envisagé par Höller comme une mise en disposition à revers d'une mise en forme, se substitue ici à une "logique de position " une "logique de mouvement ${ }^{11}$ " plus souple et perméable où les trajets et leurs connexions priment sur les principes de stabilité, d'identité ou de propriété.

\section{Porosités spatiales}

6 C'est également un espace glissant que Gilles Barbier ${ }^{12}$ nous donne à voir lorsqu'il expose en 2009 une pièce intitulée La patinoire ${ }^{13}$. Sur une large et mince palette elliptique en plexiglas, surface blanche et lisse posée en biais à même le sol, quantité de petites sculptures se culbutent: mottes de margarine et camemberts fondus, bananes, fragments de cervelle baignant dans leur jus, vers blancs écrasés, guacamole et haricots en sauce, déjections. Le geste passe ici essentiellement par ses traces après-coup : au gré des courbes dispensées par chaque objet patineur, vert et rose, jaune orange et brun entrent joyeusement en collision. Les itinéraires se croisent et se superposent par giclures, tantôt incurvés, tantôt en tourbillons. Cette chorégraphie qui réunit les différents « agents mouillants » présents dans l'œuvre de Barbier témoigne avant tout d'une création volontiers orientée vers la " vaseline onirique ${ }^{14}$ ", soit une entité flexible et proliférante qui endosse plusieurs aspects, et fonctionne chez l'artiste comme un lubrifiant conceptuel. Mais s'y déploie aussi un milieu comestible aux limites indécidables, dépourvu de motif privilégié, et dont la surface épidermique affecte les denrées autant que les locomotions. Chaque élément, comme excessivement huilé, entre en contiguïté avec son voisinage immédiat, et ce faisant, se désunit.

7 L'espace de La patinoire est régi par une interpénétration qui évoque le corps du consommateur contemporain, chez qui le système digestif, soudain médiatisé par le passage incessant de l'information, devient «extériorisé et horizontalisé ${ }^{15}$ »-le lien entre consommation et glissade relève notamment de l'observation par l'artiste des toboggans installés systématiquement aux abords des fast foods, et qu'il apparente à de gros intestins colorés ${ }^{16}$. Il s'agit d'un corps vorace (les mets cuisinés, la figure du ver 
dans son rapport digestif au réel, les dépôts de merde), ultra mobile (des peaux de banane aux beurres luisants), ouvert à toutes les flexibilités (la matière grise, le fromage mou et dégoulinant). Dans La patinoire, ce lieu paradigmatique du divertissement consumériste, les organes s'étalent et se décomposent sans plus chercher à rassembler leurs bouts. Leur force est de faire de cette dépense excessive, de cette dépossession, un nouveau mode de locomotion, impur et dépourvu de monopole. Il est question d'une explosion d'ordre schizophrénique qui s'assimile - toujours selon l'artiste - à un trauma post-opératoire : les corps s'éparpillent, excédés par l'abandon d'une forme fixe. Par cette texture grouillante d'aliments qui s'enchevêtrent et coagulent, s'imbriquent et dérapent, la glissade est le vecteur d'une porosité entre intérieur et extérieur, corps et environnement, privé et public: elle traduit l'être exposé d'une circulation universelle des signes, la prédilection contemporaine pour le packaging, ce "dedans transformé en dehors ${ }^{17}$ » dans un comble de visibilité. Elle offre autant de déclinaisons du corps dans son stade médiatique, devenu publicité de luimême, et à travers lequel « la peau n'est plus une limite mais bien une porte qui s'ouvre et autorise le passage de l'information ${ }^{18} »$. Dans cette chair en transit, glissante et traversable, potentiellement tout circule.

8 Les particularités d'un geste contemporain de glissade se précisent si on l'interroge en regard de productions antérieures. Le titre anglais de l'installation de Barbier, The Rink $^{19}$, peut en effet nous renvoyer à un court-métrage de Charlie Chaplin daté de 1916. Dans cet opus tourné pour la Mutual, nous retrouvons Charlot en serveur effronté et séducteur, usant de son habileté hors pair en patins à roulettes pour conquérir une jeune femme. Si glisser permet ici à Chaplin d'esquiver ses différents rivaux en les entraînant dans de multiples chutes, ce geste a d'abord le sens d'une distinction à la fois physique et sociale ${ }^{20}:$ c'est par lui que le personnage Charlot se rend possesseur du monde qui l'entoure et assoit sa centralité (tout tourne autour de lui). Tout en faisant écho au cinéma burlesque, l'espace de La patinoire s'offre davantage comme une gigantesque soupe où les trajets priment sur les destinations, et où la chute, entendue aussi dans le sens d'une résolution, s'aplatit pour faire prévaloir les intersections. La multitude des matières en déconfiture y provoque une déhiérarchisation autant qu'une dépersonnalisation du mouvement: nous circulons visuellement des unes aux autres sans pouvoir isoler une mimique qui leur serait propre ; le "sujet patineur" censé avoir déclenché la chorégraphie des substances au sol a disparu. Comme si la fluidité gestuelle de Charlot, qui entrait quant à elle en contraste avec la locomotion maladroite des autres personnages, concernait désormais l'espace dans son entier, espace dans lequel les éléments pris de mollesse n'occupent aucune place, ou plutôt occupent une place variable et sans personne.

9 Il faut souligner ici le rapport incoercible entre le gag burlesque traditionnel et son personnage $^{21}$ : à une silhouette reconnaissable entre toutes (Chaplin muni de sa célèbre canne, chapeauté et agile) s'adjoint une gestuelle spécifique qui lui confère une manière d'exister, y compris dans la variété de l'improvisation. Ainsi chaque acteur se dote-t-il d'une figure comique relativement circonscrite - Keaton relate d'ailleurs qu'il échangeait fréquemment ses idées de gag avec Chaplin ou Lloyd, en fonction de leurs profils respectifs. C'est ce lien même qui semble s'amenuiser au sein des corps dérapés qui se projettent sur La patinoire, explosant en saucées (et peut-être pourrait-on en dire autant en ce qui concerne les utilisateurs des toboggans de Carsten Höller, tant la vitesse de la propulsion porte atteinte aux contours de la silhouette: les clichés photographiques de cette œuvre comportent systématiquement une part de flou). D'un 
geste de détachement encore unifié bien que turbulent (celui de Chaplin), on passe alors à une dispersion dépourvue d'ancrage solide comme de schéma directeur. En d'autres termes, nous sommes en présence de produits dérivés aux qualités optionnelles, plus interchangeables.

\section{Faire geste de dé-posture}

10 En tant que posture mouvante, glisser semble mal s'accommoder des ciblages identitaires et des valeurs enracinées, et se détache dans le même temps de considérations esthétiques associées aux notions de génie ou d'autorité exclusive. Suivant un mouvement général de dilatation (du copyright, de l'individu comme entité close ou somme de qualités définies), la glissade est par là aussi, peut-être, un geste sans style - notons à ce titre que les artistes convoqués ici développent une production qui ne saurait s'assimiler ni à l'emploi d'un médium spécifique, ni à une originalité au sens où l'emploie Rosalind Krauss dans son exploration des mythes modernistes, soit comme "parabole de l'auto-création absolue ${ }^{22}$ ». Fredric Jameson a brillamment analysé combien l'essor de la « logique culturelle du capitalisme tardif » coïncidait avec une remise en cause de la métaphysique de l'intérieur et de l'extérieur, et ce faisant, de l'esthétique moderne de l'expression et du style. Les modèles de profondeur et de solidité, emblématisés chez lui par les souliers de paysan peints par Van $\mathrm{Gogh}^{23}$, laissent place à une platitude paradoxalement euphorique : celle des chaussures sérigraphiées par Warhol - auquel les bananes en cire de Gilles Barbier, notamment dans La patinoire, font explicitement référence.

11 Soulignant la complexité interprétative qui émane des objets relationnels qui nous entourent, l'impossibilité d'un décodage statique et la nécessité d'un réajustement constant à leur égard, Jameson compare ce phénomène à une «nouvelle machine, qui ne représente pas le mouvement, comme l'ancienne machinerie moderniste de la locomotive et de l'avion mais [...] n'est susceptible que d'une représentation en mouvement ${ }^{24}$ ». Par le jeu en surface qu'il implique, glisser participe d'un changement du statut gestuel - l'on est glissé plus que l'on ne glisse? - et s'envisage comme une location continue du sens et des options à prendre. La logique de propriétééc, celle des territoires permanents, s'y trouve supplantée, tandis que l'interpénétration des positions, l'emprunt de qualités multiples y constituent un facteur majeur qui remet profondément en cause les dualismes par le biais desquels on a coutume de penser le corps social comme l'activité artistique. Mais si la glissade témoigne d'un geste désorienté, opérant au sein d'une marchandisation générale et de ses interminables transits, il n'en demeure pas moins que son usage au sein des pratiques contemporaines peut aboutir à l'élaboration de scénarios alternatifs qui déconstruisent les étiquetages. Le déficit de cartographie, pris à revers d'une situation d'indistinction paralysante, devient alors un geste de dé-posture qui s'efforce justement de ne pas se correspondre.

Lorsqu'en 1991, Fabrice Hyber $(\mathrm{t})^{26}$ présente à la première biennale de Lyon le plus gros savon jamais fabriqué, intitulé Traduction, il met en place une réflexion inédite sur les rapports de l'art avec l'entreprise. Promenée de province en province par camion, dans la benne aux pans amovibles qui fut son moule d'origine, cette masse de vingt-deux tonnes s'expose sur les parkings Leclerc et se diffuse par brochures, conformément au contrat passé par l'artiste avec la compagnie agroalimentaire. Non seulement la pièce produite ne se contente pas d'être une sculpture (qui oscillerait, formellement, entre le 
quadrilatère minimaliste et le microrelief odorant), mais elle se conçoit comme une proposition itinérante issue d'un partenariat (la traduction désignant de ce point de vue autant un transport - un principe de mobilité - qu'une trahison - vis-à-vis des structures traditionnelles du marché de l'art). Sur le mode d'un échange équitable (la production de l'œuvre et sa campagne de communication avantagent les deux partis), Hyber développe une conception du commerce qui entend renouer avec le matérialisme des contacts à échelle humaine : la masse savonneuse, qui ne dépasse pas le niveau de l'œil (elle n'écrase pas le regardeur malgré son volume) est aussi une affaire de consistance; elle vient réinjecter du palpable, un volume parfumé, par opposition à une économie abstraite en rupture avec le réel.

Via ce commerce, l'artiste aspire parallèlement à « assouplir nos limites par mélange d'humeur ${ }^{27} »$ : on retrouve une fois de plus l'idée d'une perméabilité, et il n'est pas anodin qu'un tel échange passe précisément par la matière savon comme un moteur propice à tous les glissements. Par ailleurs, le décloisonnement concerne ici autant les compétences des partenaires impliqués, leur variabilité, que la transformation active des données identitaires, Hyber assimilant volontiers Traduction à un autoportrait fuyant :

Je voulais montrer que j'étais impossible à cerner; le plus impossible à cerner du monde. Cerner évoque pour moi la notion de cible sur laquelle on tire. Je ne suis pas une cible. Et je ne veux pas être limité à quelque chose. J'ai donc imaginé ce qu'il pouvait y avoir de plus incernable au monde, et quoi de plus incernable qu'une savonnette qui glisse des mains tout le temps ?28

Dans cette optique, Traduction nous enjoint de nous projeter, via une matrice de réalités, au point de départ d'extensions multiples, comme un moule d'entreprise à rebours. Glisser revient désormais à refuser l'économie du listage et des profils-types à atteindre : à cela, Hyber oppose l'idée d'un individu furtif car non défini, et de ce fait, difficilement contrôlable.

\section{Les déviations possibles}

Le surfeur sur sa vague, à l'aise dans ses tubes d'eau flexibles, ne saurait être associé exclusivement à un comble de malléabilité. Au vu de ces quelques œuvres, glisser peut même s'envisager comme un geste de résistance souple. Car dès lors que l'on s'attache à la considérer en marge des flux normatifs et de leur passivité supposée, la glissade possède des ressources qui l'allient à d'autres gestes: ceux de la déviation, de l'infiltration (se glisser) ou de la mise en frottements. Prenons un exemple qui concerne aussi bien le déplacement spatial que temporel, l'espace urbain qu'une lecture renouvelée des œuvres modernes : avec Riding Modern Art ${ }^{29}$, en 2007, Raphaël Zarka ${ }^{30}$ fait dialoguer une collection de onze photographies noir et blanc - dans lesquelles des sculptures publiques se voient prises d'assaut par des skateurs - avec une pièce de la constructiviste polonaise Katarzyna Kobro (Spatial composition 3, 1928) présentée sur son socle. Cette dernière, dont la structure géométrique épurée et l'arc inversé peuvent renvoyer à une rampe de skate, se trouve comme réactivée par les clichés photographiques. De même, les figures acrobatiques réalisées par les riders à partir des monuments publics leur insufflent une dynamique nouvelle : redevenus des étendues praticables, ces lieux et ces formes historiquement datées s'ouvrent à de nouveaux usages et potentiels transversaux. 
16 Le geste agile des skateurs vient témoigner d'une réappropriation par la glisse qui engage à la fois les œuvres elles-mêmes et l'espace attenant. Par ce réinvestissement, il s'agit de dégager un terrain de jeu qui passe par le skateboard comme pratique des différents «types de frottements » : ainsi, "pour eux [les skateurs], tout l'intérêt d'une sculpture tient à la variété des mouvements qu'elle suggère. Cette pratique de l'œuvre d'art souligne de manière irrévérencieuse, avec le vandalisme que cela suppose, le dynamisme implicite d'un grand nombre de sculptures modernes ${ }^{31} »$. On voit bien en quoi le geste de glissade acquiert ici une dimension temporelle : le déplacement des skateurs participe à réintroduire du mnésique en interrogeant dans le même temps la relation ambivalente qui relie l'œuvre de Zarka aux parangons modernistes. Glissant sur ses propres modèles, l'artiste s'en défait (la dissolution des fantasmes de pureté) précisément parce qu'il s'en remémore (l'exigence de dérèglement). La glisse, en déstabilisant les formes, en les ôtant du confort dans lesquelles elles étaient unanimement plongées (sans l'intervention des skateurs, ces œuvres se fondaient avec indifférence dans le paysage) rejoint alors le geste sculptural pour engendrer une expérience esthétique à part entière ${ }^{32}$ : elle devient à ce titre, et paradoxalement, une mise à l'épreuve du lisse.

La manière dont Deleuze et Guattari ont pensé la distinction et l'interrelation entre espace lisse et espace strié nous semble utile pour prendre acte des circulations impliquées par la glissade, dans ce qu'elle comporte d'écueils mais aussi d'ouvertures. Ainsi le geste de glisser se lie-t-il à l'espace nomade (lisse), qui « occupe l'espace sans le compter " par projections accidentées du devenir, quand l'espace sédentaire (strié), davantage métrique et rectiligne, s'applique à définir des propriétés stables en vue d'une organisation raisonnée "compt[ant] [l'espace] pour l'occuper ${ }^{33}$ ». C'est le geste emporté et variable du "suivre " par opposition à l'extériorité fixe du "reproduire ». Toutefois, ces deux types ne cessent de s'enchevêtrer, les striages se muant parfois en fluidités (à l'image d'un espace capitaliste donnant dans le flexible), et inversement, les tâtonnements du lisse étant sans cesse récupérés par l'érection de constantes (les divers fixatifs qui encadrent l'expérimentation). De fait, chacune des œuvres que nous avons abordées oscille entre ces pôles et met en jeu des tensions irrésolues : le lisse relève de l'hydraulique, des consistances fluides perpétuellement en passage sur des plans inclinés, mais le strié s'empresse de le canaliser en le subordonnant à des conduits - les toboggans d'Höller, tour à tour figures de tests empiriques ou de conductions dirigées. Les prototypes de l'espace lisse que sont la mer, le désert ou la glace $^{34}$, ces lieux de navigations ouvertes, tentent d'échapper aux cartographies qui les quadrillent - les corps patineurs de Barbier dans ce qu'ils impliquent à la fois de porosités enjouées et de conditionnement consumériste. L'important en termes de glissade étant peut-être de rendre les déviations possibles, et ce en ne cédant ni à la clôture territoriale, ni à l'emprise directionnelle - Hyber choisissant l'espace a priori strié de l'entreprise pour produire un anti-portrait qui met en fuite les ciblages, ou Zarka débordant l'assise monumentale par l'entremise des frottements du skateboard.

18 Au-delà d'une indistinction vécue comme sclérosante ou dangereusement consensuelle, la glissade se dote parfois d'une labilité qui lui permet de déconstruire les antinomies trop tranchées et de réintroduire des vecteurs dynamiques - c'est là bien souvent que l'humour intervient, moins comme une tentative de collision frontale qu'en un pas de côté très dansé (le glissé). Lorsque Fischli \& Weiss s'appliquent à ausculter les Opposés populaires qui structurent nos systèmes de pensée (Suddently this Overview, 1981-2006), 
dans une série de petites sculptures d'argile non cuite, ils font précisément appel à la figure du skieur pour malmener le binôme possible/impossible : un personnage muni de skis parvient à passer au travers d'un sapin, et les traces qu'il laisse au sol se poursuivent en continu, opérant un écart qui permet miraculeusement le passage. Par le biais d'un geste qui n'est pas une interruption mais une simple déviation de trajectoire, on trouve une promotion du déplacement qui n'ignore pas l'obstacle mais le contourne de part et d'autre pour s'en dégager. Ceci est pourtant tout le contraire d'une équivalence marchande qui reviendrait à confondre absolument les termes entre eux: les tensions demeurent perceptibles, tandis que les limitations (physiques, conceptuelles, politiques) sont rendues inopérantes en pariant sur le fait que toute configuration, quelle qu'elle soit, peut être déplacée.

Pour peu qu'un geste puisse imprégner la topographie d'une époque, la translation opérée par la glissade s'inscrit dans une interactivité et une instabilité qui lui confèrent moins un lieu et un sens propres qu'une texture à la fois souple et singulière. La locomotion des corps en milieu fluide, suivant des proximités approximatives, malmène les coordonnées gestuelles. Mais s'associer ainsi aux surfaces lisses qui l'environnent n'ampute pas nécessairement le geste de toute efficacité, même paradoxale: au sein des œuvres abordées ici, le mouvoir se décentre et se manque constamment comme cible, puisqu'il ne cesse de déraper ; il constitue pourtant un outil de diversion des striages qui persistent dans les flux, tout en composant avec l'apparente ductilité qu'ils entendent aujourd'hui revêtir. En débordant les cadres d'une dose non négligeable d'indétermination, glisser s'entend alors comme un défi : celui d'une création par dé-position, à la fois alerte et mobile.

\section{BIBLIOGRAPHIE}

Boltanski Luc, Chiapello Ève, Le nouvel esprit du capitalisme, Paris, Gallimard, 1999.

Caillois Roger, Les jeux et des hommes, Paris, Gallimard, 1958.

Castells Manuel, L'ère de l'information, vol. I, La société en réseaux [The Rise of Network Society, 1996], Philippe Delamare (trad.), Paris, Fayard, 2001.

Deleuze Gilles, Logique du sens, Paris, Minuit, 1969.

Deleuze Gilles, Guattari Felix, Mille plateaux, Paris, Minuit, 1980.

Dreux Emmanuel, Le cinéma burlesque ou la subversion par le geste, Paris, L'Harmattan, 2007.

Hal Foster, Design \& Crime [Design and Crime, and other Diatribes, 2002], Christophe Jaquet, Laure Manceau, Gauthier Herrmann et Nicolas Vieillescazes (trad.), Paris, Les prairies ordinaires, 2008. Jameson Fredric, Le postmodernisme ou la logique culturelle du capitalisme tardif [Postmodernism or The Cultural Logic of Late Capitalism, 1991], Florence Nevoltry (trad.), Paris, Éd. de l'ENSBA, 2007.

Krauss Rosalind, «L'originalité de l'avant-garde : une répétition postmoderniste », in L'originalité de l'avant-garde et autres mythes modernistes [The Originality of the Avant-garde and Other Modernist Myths, 1985], Jean-Pierre Criqui (trad.), Paris, Macula, 1993. 
Marcadé Bernard, « L'art, c'est toutes les possibilités du monde », in Hyber, Paris, Flammarion, 2009.

Mongin Olivier, Éclats de rire, variations sur le corps comique, Paris, Seuil, 2002.

Semprini Andrea, La société de flux, formes du sens et identité dans les sociétés contemporaines, Paris, L'Harmattan, 2003.

Sterckx Pierre, Gilles Barbier, un abézédaire dans le désordre, Paris, Éditions du Regard, 2008.

Wetterwald Élisabeth, «Entretien avec Raphaël Zarka », 20/27, nº 3, Paris, 2009.

Zarka Raphaël, Une journée sans vagues, chronologie lacunaire du skateboard, Paris, Éditions F7, 2006.

Zarka Raphaël, La conjonction interdite, notes sur le skateboard [2003], Paris, Éditions F7, 2007.

\section{NOTES}

1. «Gilles Barbier, le monde est une tong ", entretien avec Richard Leydier in Artpress, $n^{\circ} 368$, juin 2010, p. 34.

2. Gilles Deleuze, Logique du sens, Paris, Minuit, 1969, p. 158.

3. Ainsi, à propos des designers actuels: «Du spectacle, ils sont moins les critiques que les surfers ", Hal Foster, Design \& Crime (Design and Crime, and other Diatribes, 2002), trad. par Christophe Jaquet, Laure Manceau, Gauthier Herrmann et Nicolas Vieillescazes, Paris, Les prairies ordinaires, 2008, p. 38.

4. CEuvre visible sur http://www.airdeparis.com/artists.htm

5. Höller accompagne Test Site d'une étude de faisabilité commandée à la General Public Agency (Londres), qui examine plus avant les modalités contextuelles, économiques et sécuritaires d'une utilisation de toboggans permanents au sein de nos mégalopoles. Voir Carsten Höller, Test Site, Tate Modern, London, exposition du 10 octobre 2006 au 9 avril 2007, notamment p. 57-85.

6. Carsten Höller, Source Book, Tate Modern, London, exposition du 10 octobre 2006 au 9 avril 2007, sélection de textes et préface de l'artiste, p. 6.

7. «I feel that the frequent use of slides leads to a particular form of change ", Ibid., p. 5. Dans ce même ouvrage, Höller se réfère explicitement aux écrits de Roger Caillois (Les jeux et des hommes, Paris, Gallimard, 1958).

8. À propos du « phénomène Valerio », Ibid., voir p. 69-71.

9. Manuel Castells, L'ère de l'information, vol. I, La société en réseaux (The Rise of Network Society, 1996), trad. par Philippe Delamare, Paris, Fayard, 2001, voir notamment p. 510 et sq.

10. Cette notion, empruntée à l'origine aux sciences de la nature, fut réactualisée par l'essor de l'électronique dans les années 1960-1970. Sur les propriétés du flux que nous reprenons ici en partie, nous renvoyons à l'ouvrage d'Andrea Semprini, La société de flux, formes du sens et identité dans les sociétés contemporaines, Paris, L'Harmattan, 2003, notamment p. 100-106.

11. Ibid., p. 90.

12. http://www.galerie-vallois.com/artistes/gilles-barbier.html

13. Installation présentée du 26 juin au 18 octobre 2009 à la Villa Arson, Nice.

14. Cette expression, empruntée à Gilles Barbier, est entre autres l'intitulé d'une sculpture datée de 2003 : la réplique en cire d'une peau de banane jonchant le sol.

15. Gilles Barbier cité in Pierre Sterckx, Gilles Barbier, un abézédaire dans le désordre, Paris, Éditions du Regard, 2008, p. 65.

16. Voir l'entretien «Before anything else... a conversation beetween Jean-Yves Jouannais and Gilles Barbier » in Gilles Barbier, Paris, JPR/Ringier, 2005, p. 71.

17. Gilles Barbier cité in Pierre Sterckx, Gilles Barbier, un abézédaire dans le désordre, p. 260-261. 
18. Ces annotations figurent dans la gouache intitulée Sans titre (La conquête de l'espace), en 2003.

19. Nous renvoyons à la gouache intitulée Wetting Agents, en 2009.

20. Comme l'a souligné Olivier Mongin, il s'agit pour Chaplin l'émigrant de « se faire une place ", tout en remettant «les choses à leur place» par le biais d'un retournement. Olivier Mongin, Éclats de rire, variations sur le corps comique, Paris, Seuil, 2002, voir p. 23 et sq.

21. À ce sujet, voir l'étude d'Emmanuel Dreux, Le cinéma burlesque ou la subversion par le geste, Paris, L'Harmattan, 2007, p. 83.

22. Rosalind Krauss, "L'originalité de l'avant-garde: une répétition postmoderniste", in L'originalité de l'avant-garde et autres mythes modernistes (The Originality of the Avant-garde and Other Modernist Myths, 1985), trad. par Jean-Pierre Criqui, Paris, Macula, 1993, p. 135.

23. Fredric Jameson, Le postmodernisme ou la logique culturelle du capitalisme tardif (Postmodernism or The Cultural Logic of Late Capitalism, 1991), trad. par Florence Nevoltry, Paris, Éd. de l'ENSBA, 2007, voir notamment p. 40-55.

24. Ibid., p. 93.

25. À ce propos, voir aussi l'étude sociologique de Luc Boltanski et Ève Chiapello, Le nouvel esprit du capitalisme, Paris, Gallimard, 1999, notamment p. 232 et sq. Certains travaux de Gilles Barbier, dont Emmental Head (2003) tournent explicitement autour des notions de location et de propriété en regard du buste de l'identité.

26. http://www.hyber.tv/

27. Hyber cité in Bernard Marcadé, «L'art, c'est toutes les possibilités du monde », Hyber, Paris, Flammarion, 2009, p. 58.

28. Ibid., p. 41.

29. Présentée à la biennale de Lyon de la même année, cette œuvre fait suite à une vidéo du même nom datée de 2005.

30.

http://www.michelrein.com/Artist.php?

Artist=Rapha\%C3\%ABl\%20Zarka\&Artist_Search_page $=4$

31. Élisabeth Wetterwald, « Entretien avec Raphaël Zarka », 20/27 n 3, Paris, m19, 2009, p. 273.

32. Voir à ce titre les deux opus que l'artiste a consacré au skate : La conjonction interdite, notes sur le skateboard (2003), Paris, Éditions F7, 2007, et Une journée sans vagues, chronologie lacunaire du skateboard, Paris, Éditions F7, 2006.

33. Gilles Deleuze et Felix Guattari, Mille plateaux, Paris, Minuit, 1980, p. 447. Les termes sont repris à Pierre Boulez.

34. Ibid., voir p. 481, p. 598-599. Rappelons ici qu'étymologiquement, « glisser » vient du mélange de « gliier » (ancien français) et de « glacer ».

\section{RÉSUMÉS}

En regard de quelques œuvres contemporaines qui font appel à la glissade à divers titres, ce texte se propose d'interroger ce que serait un geste en milieu fluide. Il s'agit d'ébaucher quels types de corps, de spatialités et de locomotions s'y déploient en écho à leur époque, mais selon une acception dynamique qui envisage le geste de glisser au-delà d'une assimilation passive. 
INDEX

Mots-clés : art contemporain, flux, geste, glisser

AUTEUR

CHARLOTTE SERRUS

Docteur en arts plastiques, Aix-Marseille université, charlotte.serrus@wanadoo.fr 